\title{
Evaluation of Gravity Data Derived from Global Gravity Field Models Using Terrestrial Gravity Data in Enugu State, Nigeria
}

https://doi.org/10.1515/jogs-2018-0015

Received February 24, 2018; accepted November 26, 2018

\begin{abstract}
Spherical harmonic expansion is a commonly applied mathematical representation of the earth's gravity field. This representation is implied by the potential coefficients determined by using elements/parameters of the field observed on the surface of the earth and/or in space outside the earth in the spherical harmonic expansion of the field. International Centre for Gravity Earth Models (ICGEM) publishes, from time to time, Global Gravity Field Models (GGMs) that have been developed. These GGMs need evaluation with terrestrial data of different locations to ascertain their accuracy for application in those locations. In this study, Bouguer gravity anomalies derived from a total of eleven (11) recent GGMs, using sixty sample points, were evaluated by means of Root-Mean-Square difference and correlation coefficient. The Root-Mean-Square differences of the computed Bouguer anomalies from ICGEM website compared to their positionally corresponding terrestrial Bouguer anomalies range from $9.530 \mathrm{mgal}$ to 37.113 mgal. Additionally, the correlation coefficients of the structure of the signal of the terrestrial and GGM-derived Bouguer anomalies range from 0.480 to 0.879 . It was observed that GECO derived Bouguer gravity anomalies have the best signal structure relationship with the terrestrial data than the other ten GGMs. We also discovered that EIGEN-6C4 and GECO derived Bouguer anomalies have enormous potential to be used as supplements to the terrestrial Bouguer anomalies for Enugu State, Nigeria.
\end{abstract}

Keywords: Bouguer Anomalies; Global Gravity field Models; International Centre for Gravity Earth Model

\footnotetext{
O.I. Apeh: Department of Geoinformatics \& Surveying, University of Nigeria, Enugu Campus, Nigeria, E-mail: ojima.apeh@unn.edu.ng, apehisaac@yahoo.com

E.C. Moka, V.N. Uzodinma: Department of Geoinformatics \& Surveying, University of Nigeria, Enugu Campus, Nigeria
}

\section{Introduction}

Terrestrial gravity observation has always produced sparse network of points where gravity have been observed. This is due to the tediousness of the methods used. Some of the uses of these gravity values such as for geoid determination require the knowledge of gravity values all over the earth. This has not been possible till date using terrestrial measurements alone. Fortunately, satellite technology has come to the rescue. It is now possible to observe gravity values from space. Interestingly, the development and enhancement in technology has continued to improve, easing the activities of humanity in general and geoscientists in particular. This has brought about improved knowledge of the dynamics of the earth. It is no longer news that a number of Global Gravity Field Models (GGMs) are being developed by different scientific research groups with increasing applications in geosciences and there is need to evaluate them using terrestrial data in order to ascertain their accuracy in some regions.

Barthelmes (2013) emphasized on the need for the evaluation of gravity field functional using terrestrial data in order to ascertain their accuracy. There are similar studies carried out in some regions of the world in which terrestrial data was used to determine the accuracy of the gravity functional derived from GGMs. In Finland, an evaluation of satellite-based GGMs, using terrestrial data, gave height anomaly differences of around $15 \mathrm{~cm}$ and gravity anomaly differences of around 10mgal (Saari \& BilkerKoivula, 2015); a comparative analysis of some satellitebased GGMs carried out in Norway gave several mgal of gravity anomalies (Šprlák et al., 2011); while a similar study done in Canada gave the RMS difference between gravity anomalies derived from GGM and terrestrial gravity data as $5 \mathrm{mgal}$ on sea and lake surfaces but $14 \mathrm{mgal}$ was observed on land meaning that the RMS indicates a height-dependent trend (Huang et al., 2009); In Kenya, an evaluation of free-air gravity anomaly derived from EGM2008 gave a standard deviation of \pm 10.7 mgal (Odera, 2016 ); In Turkey, an evaluation of GGM-derived geoid 
heights with GPS/leveling derived geoid heights revealed that EGM2008 best fits $( \pm 0.157 \mathrm{~m})$ to the GPS/levelling derived geoid heights (Yilmaz et al., 2010).

Global Gravity Field Models are required for such applications as orbit determination, inertial navigation, deformation monitoring, geoid undulation computation, oceanographic applications, and geophysical prospecting, among others. They are given in form of sets of spherical harmonic coefficients. The recently developed models are improvements over the earlier ones and tend to be more accurate as illustrated in International Centre for Global Earth Models (ICGEM) website (Barthelmes \& Köhler, 2016). A GGM is normally validated using gravity field data that was not used in the computation of the GGM. The deviations of the directly observed values from the model derived quantities will determine the confidence to be placed in the use of the GGM in any particular area and for different applications. The Bouguer gravity anomalies obtained from these GGMs can be used in modelling the gravimetric geoid, in geophysical prospecting, in engineering works, in simulation studies, in geodynamics studies like crustal movement and a host of other applications.

Owing to the usefulness of Bouguer gravity anomalies in many applications and as a result of recent publications of GGMs, this study aimed at evaluating the accuracy of the Bouguer gravity anomalies derived from eleven (11) GGMs using terrestrial Bouguer anomalies so that where necessary they could be used in place of or in combination with directly observed values in Enugu State, Nigeria.

\section{Terrestrial Gravity Data, GGMs and Methodology}

\subsection{Terrestrial Gravity Data}

Nigeria Geological Survey Agency (NGSA) provided the terrestrial Bouguer gravity data used in this research. Thirtysix evenly distributed gravity base stations were established across the entire State. A gravity base station at Nkalagu was used as well as the base station at the General Post-Office Enugu as controls. This third-order gravity survey was tied to IGSN'71 (Morelli et al., 1972) through PGNN (Osazuwa,1986). As published by NGSA, the following instruments were used for the terrestrial observation of Bouguer gravity data in Enugu State.

1. Lacoste and Romberg (G-512) gravimeter $( \pm 0.01$ mgal)

2. FA-181 Wallace, Tiernan and Brunton Barometric altimeters $( \pm 1 \mathrm{~m})$
3. American Paulin System MDM- $5( \pm 0.5 \mathrm{~m})$

4. Sling Psychrometer

5. Garmin Csx 76 GPS $( \pm 3 \mathrm{~m})$

The Gravimeter was calibrated using Northern Nigeria Calibration Line (Jos-Ilela) -376.72 mgal (Osazuwa,1992a). The scale was calibrated to the Smithsonian meteorological table and can be read to $10 \mathrm{ft}$ ( $3 \mathrm{~m}$ ) accuracy. The sling psychrometer was used to measure the Air temperature while the relative humidity used in correcting the barometric readings was determined from the psychrometric chart.

Bench marks (obtained from leveling) of known heights were obtained from the then Federal Surveys office (now Office of the Surveyor General of the Federation (OSGoF)) in Enugu State and were used as controls in the determination of elevations. Even though these benchmarks may not necessarily be gravity stations, however the altimeters were read at all the known heights with the points looped into the survey for proper monitoring of drift of the Altimeters.

Heights of gravity stations were measured with two FA181Wallace \& Tiernan and one Brunton altimeters. These instruments are essentially precision aneroid barometers with low-friction mechanical amplification to the dial. The altitude correction chart combined the correction factors for air temperature and relative humidity as they appear respectively in Tables 52, and 54 of the fifth revised Edition of the Smithsonian meteorological tables. The heights of the gravity stations were therefore derived from the average results of the two Wallace and Tiernan altimeters which were observed, at least once at each station and also at the station of the primary gravity network.

The common corrections applied in this gravity survey include latitude, tidal, altitude, free air, Bouguer, terrain and drift corrections. The refined Bouguer Anomaly is obtained thus:

$$
B A=G^{o b s}-G_{t h}+F-B_{c}+T_{c}
$$

where: $G^{o b s}=$ Observed Gravity, $G_{t h}=$ latitude correction, $B A=$ Bouguer Anomaly, $B_{c}=0.1119 H=$ Attraction of Bouguer plate, $F=0.3086 \mathrm{H}=$ Free air reduction, $T_{C}=$ Terrain correction. For this survey, 1967 Geodetic Reference System Gravity formula was used.

$$
\begin{aligned}
& G_{t h}=978031.85\left(1.0+0.005278895 \sin ^{2} \varphi\right. \\
& \left.+0.000023462 \sin ^{4} \varphi\right)(\mathrm{mgal})
\end{aligned}
$$

where: $\varphi=$ Latitude.

The resulting Bouguer anomalies as obtained and provided by NGSA were boxed into grid cells to check for sudden jumps or discontinuities. The result of Bouguer 
anomalies as obtained by the Nigerian Geological Survey Agency for the area of study is shown in Fig. 1

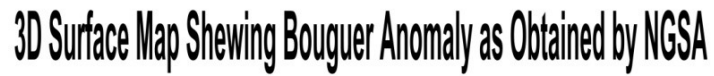

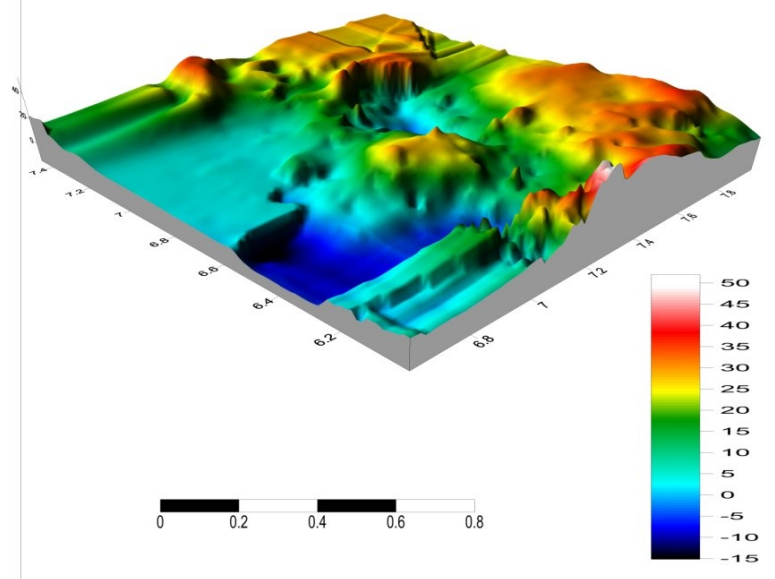

Figure 1: 3D Surface map of terrestrial Bouguer anomaly

The result of this survey shows a good correlation between the Bouguer anomalies and the surface geology of Enugu State when compared with the existing geological map. (http://www.ngsa-nig.org/content/regional-gravitysurvey-enugu-state)

\subsection{Global Gravity field Models (GGMs) Used in this Research}

The characteristics of the GGMs used in this research are shown in Table 1. These eleven (11) GGMs consist of six (6) Satellite-based Models, three (3) combined Satellite, Gravity data and Altimetry data Models and two (2) Gravity Observation Combination (GOCO) Models.

The different GGMs are characterised based on the input data, method of calculation, the degree and order of expansion which determine the resolution as well as other things modelled.

\subsection{Methodology}

Generally, the gravitational potential of the earth can be represented outside the masses of the earth by a set of fully normalized spherical harmonic coefficients $\bar{C}, \bar{S}$, called a global gravity field model. The gravitational potential, $\mathrm{V}_{E}$, can be expanded into a series of fully normalized solid spherical harmonics outside the earth as (Wenzel, 2005);

$$
\begin{aligned}
& V_{E}(r, \theta, \lambda)= \\
& \frac{G M_{E}}{r} \sum_{l=0}^{l_{\max }}\left(\frac{a}{r}\right)^{l} \sum_{m=0}^{l}\left(\bar{C}_{l, m} \cos m \lambda+\bar{S}_{l, m} \sin m \lambda\right) \bar{P}_{l, m}(\cos \theta)
\end{aligned}
$$

where; $G M_{E}=$ Earth's gravitational constant, a = semimajor axis of the reference ellipsoid, $\bar{C}_{l, m} \bar{S}_{l, m}=$ fully normalized spherical harmonic coefficients, $\bar{P}_{l, m}=$ fully normalized associated Legendre functions of first kind, $l=$ degree, $m=$ order, $r=$ radius(geocentric distance), $\lambda=$ spherical longitude, $\theta=$ polar distance (spherical colatitude), $l_{\max }=$ maximum degree of expansion

The formula for expansion of gravity anomaly in series of spherical coordinates is given in Eq. 4 (Wenzel, 2005);

$$
\begin{aligned}
\Delta g_{s a}= & \frac{G M}{r^{2}} \sum_{l=2}^{l_{\max }}\left(\frac{a}{r}\right)^{l}(l-1) \sum_{m=0}^{l}\left(\Delta \bar{C}_{l, m} \cos m \lambda\right. \\
& \left.+\Delta \bar{S}_{l, m} \sin m \lambda\right) \bar{P}_{l, m}(\cos \theta)
\end{aligned}
$$

where; $\Delta g_{s a}=$ Gravity anomaly in spherical approximation, $G M$ =Earth's gravitational constant, $\Delta \bar{C}_{l, m}=\bar{C}_{l, m}$ $\bar{C}_{l, m}^{N}, \Delta \bar{S}_{l, m}=\bar{S}_{l, m}-\bar{S}_{l, m}^{N}$.

The spatial wavelength and resolution of gravity field parameters computed from a global gravity field model is directly linked to the maximum degree and order of expansion. As a rule of thumb, the spatial resolution and the minimum wavelength are (Ibid);

$$
\begin{gathered}
\text { SpatialResolution }=\frac{20000 \mathrm{~km}}{l_{\max }}=\frac{\pi R}{l_{\max }} \\
\text { MinimumWavelength }=\frac{40000 \mathrm{~km}}{l_{\max }}=\frac{2 \pi R}{l_{\max }}
\end{gathered}
$$

where; $\mathrm{R}=6371 \mathrm{~km}=$ Mean radius of the earth

The simple Bouguer anomaly is obtained from Eq. (7) below:

$$
\Delta g_{B}(\lambda, \phi)=\Delta g_{c l}(\lambda, \phi)-2 \pi G \rho H(\lambda, \phi)
$$

where; $\Delta g_{B}=$ Bouguer gravity anomaly, $\rho=$ constant density, $\Delta g_{c l}=$ Classical gravity anomaly, $G=$ Gravitational constant, $\lambda, \phi=$ Ellipsoidal longitude and latitude.

The classical gravity anomaly is the magnitude of the downward continued gravity, $\left|\nabla W^{c}\right|$, on to the geoid minus the normal gravity, $|\nabla U|$, on the ellipsoid at the same ellipsoidal longitude, $\lambda$, and latitude, $\phi$.

$$
\Delta g_{c l}(\lambda, \phi)=\left|\nabla W^{c}(N, \lambda, \phi)\right|-|\nabla U(0, \phi)|
$$

where;

$$
W^{c}(h, \lambda, \phi)=W_{a}^{c}(h, \lambda, \phi)+\Phi(h, \phi)
$$


Table 1: Summary of the characteristics of the eleven GGMs used in this research

\begin{tabular}{|c|c|c|c|c|}
\hline $\operatorname{Model}(\mathrm{d} / \mathrm{o})$ & Symbol & Resolution & Source of Data & References \\
\hline HUST-Grace2016s(160) & $\mathrm{k}_{1}$ & $\sim 125 \mathrm{~km}$ & $\mathrm{~S}$ (Grace) & Zhou Hao et al., 2016 \\
\hline ITU_GRACE16(180) & $\mathrm{k}_{2}$ & $\sim 111 \mathrm{~km}$ & $\mathrm{~S}($ Grace $)$ & Akyilmaz et al., 2016b \\
\hline ITU_GGC16(280) & $\mathrm{k}_{3}$ & $\sim 71 \mathrm{~km}$ & S(Grace, Goce) & Akyilmaz et al., 2016a \\
\hline EIGEN-6S4v2(300) & $\mathrm{k}_{4}$ & $\sim 67 \mathrm{~km}$ & S(Goce,Grace, Lageous) & Förste et al., 2016 \\
\hline EIGEN-6C4(2190) & $\mathrm{k}_{5}$ & $\sim 9 \mathrm{~km}$ & S(Goce,Grace, Lageous), G, A & Förste et al., 2015 \\
\hline GOC005C(720) & $\mathrm{k}_{6}$ & $\sim 28 \mathrm{~km}$ & S, G, A (see model) & Fecher et al., 2016 \\
\hline GOCO05s(280) & $\mathrm{k}_{7}$ & $\sim 71 \mathrm{~km}$ & $\mathrm{~S}($ see model) & Mayer-Gürr et al., 2015 \\
\hline GGM05C(360) & $\mathrm{k}_{8}$ & $\sim 56 \mathrm{~km}$ & S(Grace, Goce), G, A & Ries et al., 2016 \\
\hline GGM05G(240) & $\mathrm{k}_{9}$ & $\sim 83 \mathrm{~km}$ & S(Grace, Goce) & Bettadpur et al., 2015 \\
\hline $\operatorname{GECO}(2190)$ & $\mathrm{k}_{10}$ & $\sim 9 \mathrm{~km}$ & S(Goce), EGM2008 & Gilardoni et al., 2015 \\
\hline GO_CONS_GCF_2_SPW_R4(280) & $\mathrm{k}_{11}$ & $\sim 71 \mathrm{~km}$ & $\mathrm{~S}($ Goce $)$ & Gatti et al., 2015 \\
\hline
\end{tabular}

$\mathrm{S}=$ Satellite Tracking Data, $\mathrm{G}=$ Gravity Data, $\mathrm{A}=$ Altimetry Data, $\mathrm{d} / \mathrm{o}=$ degree/order

$$
\begin{gathered}
W_{a}^{c}=\text { attraction potential, } \Phi=\text { centrifugal potential } \\
\qquad \Delta g_{c l}(\lambda, \phi) \approx \Delta g_{s a}(\lambda, \phi)
\end{gathered}
$$

In these GGMs, the Bouguer gravity anomaly is calculated by the spherical approximation of the classical gravity anomaly minus $2 \pi G \rho H$. The topographic heights, $\mathrm{H}(\lambda, \varphi)$, are calculated from the spherical harmonic model DTM2006 used up to the same maximum degree as the gravity field model (Barthelmes, 2013).

$$
\begin{aligned}
& \Delta g_{s a}(r, \lambda, \varphi)=\frac{G M}{r^{2}} \sum_{l=0}^{l_{\max }}\left(\frac{R}{r}\right)^{l}(l-1) \sum_{m=0}^{l} P_{l m} \\
& (\sin \varphi)\left(C_{l m}^{T} \cos m \lambda+S_{l m}^{T} \sin m \lambda\right)
\end{aligned}
$$

The topography model can be transformed into a surface spherical harmonics expansion as (Barthelmes, 2013);

$$
\begin{gathered}
H(\lambda, \varphi)=R \sum_{l=0}^{\operatorname{lmax}} \sum_{m=0}^{l} P_{l m}(\sin \varphi) \\
\left(C_{l m}^{\text {topo }} \cos m \lambda+S_{l m}^{\text {topo }} \sin m \lambda\right)
\end{gathered}
$$

where; $\mathrm{H}(\lambda, \varphi)=$ topographic Heights, $\mathrm{R}=$ Reference radius, $C_{l m}^{\text {topo }}, S_{l m}^{\text {topo }}=$ coefficients of expansion.

The calculation service of ICGEM was used for the computation of the Bouguer gravity data for the sixty (60) test points. All computations were carried out on Geodetic Reference System ellipsoid and in the Mean Tide system while the box for the zero degree term remained unchecked. The GGMs were truncated at their maximum degree of expansion and there was no filtering of any sort. Among others, it requires geodetic coordinates (Latitude, Longitude and Ellipsoidal height) of the test points to be inputted and that was what led to the acquisition of existing government geodetic control points. The ellipsoidal height is converted to orthometric height using the topography model in Eq. (12) and truncated at the maximum degree of expansion of the Global Gravity field Model (Barthelmes \& Köhler, 2016).

\section{Results}

\subsection{Statistical Results}

The values of the RMS difference show the level of accuracy of the GGM-derived Bouguer anomalies to the terrestrial Bouguer anomalies.

The values of the correlation coefficients show the structure (whether in phase or out of phase with the terrestrial data) of the signals of the GGM-derived and terrestrial Bouguer anomalies at the sixty (60) test points.

\subsection{Graphical Results}

The structure of the signal between the terrestrial and GGM-derived Bouguer anomalies are illustrated in Fig. 2.

\section{Discussions and Conclusion}

\subsection{Discussions}

The closer the value of the Root-Mean-Square (RMS) to zero, the more accurate is the GGM-derived Bouguer anomaly to the terrestrial Bouguer anomaly while the farther the value of the RMS from zero, the less accurate is 
Table 2: Statistical result of the difference between computed Bouguer anomalies and the terrestrial Bouguer anomalies

\begin{tabular}{lllllll}
\hline Model (Degree/Order) & Symbol & $\begin{array}{l}\text { MIN } \\
\text { (mgal) }\end{array}$ & $\begin{array}{l}\text { MAX } \\
(\mathrm{mgal})\end{array}$ & $\begin{array}{l}\text { MEAN } \\
(\mathrm{mgal})\end{array}$ & $\begin{array}{l}\text { STDEV } \\
(\mathrm{mgal})\end{array}$ & $\begin{array}{l}\text { RMS } \\
(\mathrm{mgal})\end{array}$ \\
\hline HUST-Grace2016s(160) & $\mathrm{k}_{1}$ & -10.175 & 23.572 & 6.687 & 7.970 & 10.440 \\
ITU_GRACE16(180) & $\mathrm{k}_{2}$ & 17.731 & 47.653 & 36.355 & 7.527 & 37.426 \\
ITU_GGC16(280) & $\mathrm{k}_{3}$ & -4.343 & 27.035 & 12.805 & 6.628 & 14.515 \\
EIGEN-6S4V2(300) & $\mathrm{k}_{4}$ & -8.467 & 30.350 & 10.550 & 8.342 & 13.519 \\
EIGEN-6C4(2190) & $\mathrm{k}_{5}$ & -1.189 & 19.165 & 8.133 & 5.013 & 9.613 \\
GOC005C(720) & $\mathrm{k}_{6}$ & -6.206 & 27.572 & 11.118 & 9.048 & 14.407 \\
GOCO05S(280) & $\mathrm{k}_{7}$ & -2.633 & 27.468 & 12.145 & 6.486 & 13.859 \\
GGM05C(360) & $\mathrm{k}_{8}$ & -9.718 & 27.563 & 7.180 & 8.246 & 10.974 \\
GGM05G(240) & $\mathrm{k}_{9}$ & -6.313 & 22.950 & 8.596 & 6.866 & 11.058 \\
GECO(2190) & $\mathrm{k}_{10}$ & 0.708 & 20.826 & 9.471 & 4.520 & 10.567 \\
GO_CONS_GCF_2_SPW_R4(280) & $\mathrm{k}_{11}$ & 0.451 & 31.801 & 16.600 & 6.329 & 17.896 \\
\hline
\end{tabular}

Table 3: Statistical result of coefficient of correlation between the terrestrial and GGM-derived Bouguer anomalies

\begin{tabular}{llc}
\hline Model (Degree/Order) & Symbol & Coefficient of Correlation \\
\hline HUST-Grace2016s(160) & $\mathrm{k}_{1}$ & 0.556 \\
ITU_GRACE16(180) & $\mathrm{k}_{2}$ & 0.646 \\
ITU_GGC16(280) & $\mathrm{k}_{3}$ & 0.730 \\
EIGEN-6S4v2(300) & $\mathrm{k}_{4}$ & 0.480 \\
EIGEN-6C4(2190) & $\mathrm{k}_{5}$ & 0.853 \\
GOCO05C(720) & $\mathrm{k}_{6}$ & 0.510 \\
GOCO05S(280) & $\mathrm{k}_{7}$ & 0.739 \\
GGM05C(360) & $\mathrm{k}_{8}$ & 0.500 \\
GGM05G(240) & $\mathrm{k}_{9}$ & 0.690 \\
GECO(2190) & $\mathrm{k}_{10}$ & 0.879 \\
GO_CONS_GCF_2_SPW_R4(280) & $\mathrm{k}_{11}$ & 0.765 \\
\hline
\end{tabular}

Table 4: Results obtained by other researchers

\begin{tabular}{llllll}
\hline S/N0 & Country & Researcher(s) & Functional & STDEV & RMS \\
\hline 1 & Finland & Saari \& Bilker-Koivula, 2015 & Gravity Anomalies & $10 \mathrm{mgal}$ \\
2 & Norway & Šprlák et al., 2011 & Gravity Anomalies & $20.7 \mathrm{mgal}$ & \\
3 & Canada & Huang et al., 2009 & Gravity Anomalies & & $14 \mathrm{mgal}$ \\
4 & Kenya & Odera, 2016 & Gravity Anomalies & $\pm 10.7 \mathrm{mgal}$ & \\
\hline
\end{tabular}

the GGM-derived Bouguer anomaly to terrestrial Bouguer anomaly. The closer the value of correlation coefficient to \pm 1 , the more accurate the structure of the signal of GGM-derived Bouguer anomaly to the terrestrial Bouguer anomaly while the farther the value of correlation coefficient from \pm 1 , the less accurate is the structure of the signal of GGM-derived Bouguer anomaly to terrestrial Bouguer anomaly at each of the test points.

The plot of the various results obtained from the GGMs with respect to the observed Bouguer gravity anomaly is an indication of the structure of the signal of the GGM-derived
Bouguer anomaly to the terrestrial Bouguer anomaly. Signal, as used in this sense, refers to the terrestrial and computed Bouguer gravity anomalies obtained at each of the given test points. Structure refers to the pattern of movements shown by both the terrestrial and computed Bouguer anomalies at each of the test points. The test points are the independent variable while the terrestrial and the computed Bouguer anomalies are the dependent variables.

The high value of the correlation coefficient (Table 3) between the computed and terrestrial Bouguer anomalies 

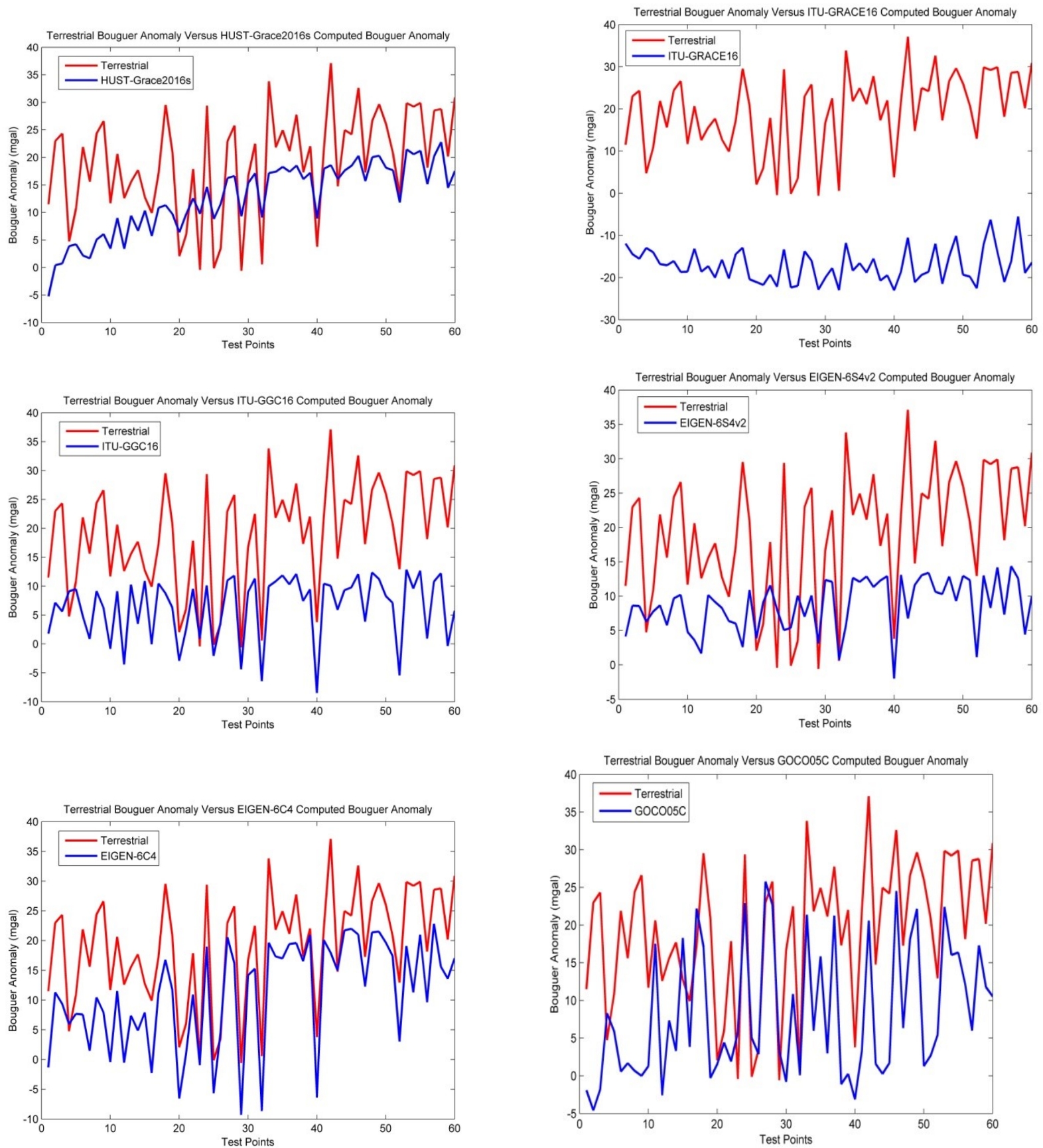

Figure 2: Graphs showing terrestrial and GGM-derived Bouguer anomalies at each test point

does not mean high level of accuracy rather it shows high level of the structure of the signals (that is, whether the computed and terrestrial Bouguer anomaly are in phase or out of phase at each of the given test points).

These eleven (11) GGMs consist of six (6) Satellitebased Models, three combined Satellite, Gravity data and Altimetry data Models and two Gravity Observation Combination (GOCO) Models. For the six Satellite-based Models, HUST-Grace16s has the least RMS (see Table 2) but GO_CONS_GCF_2_SPW_R4 shows the strongest signal structure relationship (based on the value of coefficient of correlation obtained in Table 3) between the GGM-derived Bouguer anomaly and the terrestrial Bouguer anomaly for the sixty (60) test points evaluated.

For the three combined Satellite, Gravity data and $\mathrm{Al}-$ timetry data models, EIGEN-6C4 has the least RMS (see Table 2) but GECO shows the strongest signal structure relationship (based on the value of coefficient of correlation 

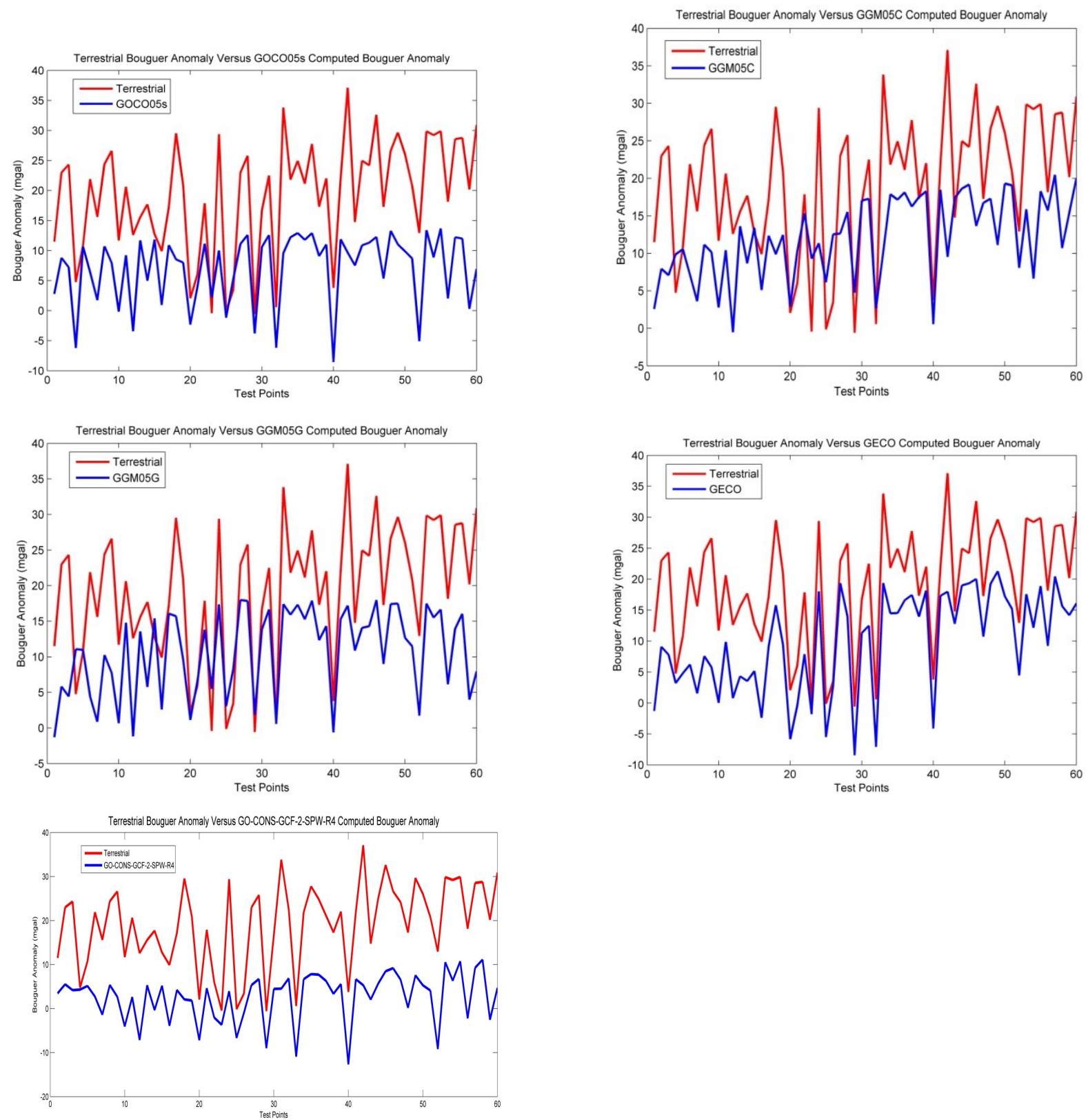

Figure 2: (cont'd) Graphs showing terrestrial and GGM-computed Bouguer anomalies at each of the given test points.

obtained in Table 3) between the GGM-derived Bouguer anomaly and the terrestrial Bouguer anomaly for the sixty (60) test points evaluated.

For the two Gravity Observation Combination (GOCO) Models, GOCO05s performed better than GOCO05C for the sixty (60) test points evaluated.

Among the eleven models evaluated, EIGEN-6C4 is the only one that has a one-digit whole number for the RMS difference (see Table 2). Comparing the eleven Global Gravity Field Models to one another based on the RMS values obtained, it can be inferred that EIGEN-6C4 is more accu- rate than the other ten GGMs which were evaluated using sixty test points in Enugu State, Nigeria.

GECO has the best signal structure relationship with the terrestrial Bouguer anomaly than the other GGMs based on the value of coefficient of correlation obtained in Table 3.

The values of Bouguer gravity data obtained from ITUGRACE16, for the sixty (60) test points, are all negative which resulted in the high value of the Root-Mean-Square and this could be partly due to the omission and commission errors inherent in the GGMs; possible systematic er- 
rors in the observed terrestrial data and the topographic bias between Digital Elevation Models (DEMs), such as ETOPO1, and the terrestrially acquired elevation data.

Standard deviations and Root-Mean-Square differences obtained in some similar studies show that the results obtained in this current study is reliable. The results obtained in Table 2 are in tandem with the results obtained by other researchers (Table 4) from other regions of the world.

The relatively large values obtained from the Root-Mean-Square (RMS) differences of the GGMcomputed/terrestrial Bouguer gravity anomalies could be partly due to the omission and commission errors inherent in the GGMs, possible systematic errors in the observed terrestrial data and the topographic bias arising from truncation of the ETOPO1 topography model (see Eq. 12) and the terrestrially acquired elevation data. This is so because there is always a bias between DEM data and terrestrial data as a result of the great deviations in gradient of the undulating terrain when point values are compared to mean values such as ETOPO1 DEM.

\subsection{Conclusion}

This study statistically evaluated the accuracy of Bouguer gravity anomalies computed from a total of eleven (11) GGMs using terrestrially observed Bouguer anomalies at sixty (60) test points in Enugu State, Nigeria. As stated earlier, the accuracy and the resolving power of the data used in the development of a Global Gravity Field Model determine its accuracy and resolution and from this study, we have discovered that EIGEN-6C4 and GECO showed enormous potential to be used as supplements to the terrestrial Bouguer gravity data. Nevertheless, improvement/remodelling/refining of these Global Gravity field Models is required to enhance their accuracy in Enugu State, Nigeria.

Acknowledgement: The authors are very grateful to the Nigerian Geological Survey Agency (NGSA) for providing terrestrial gravity data for this study.

\section{References}

Akyilmaz O., Ustun A., Aydin C., Arslan N., Doganalp S., Guney C., Mercan H., Uygur S.O., Uz M. and Yagci O., 2016a, ITU_GGC16 The combined global gravity field model including GRACE \& GOCE data up to degree and order 280. http://doi.org/10.5880/icgem.2016. 005
Akyilmaz O., Ustun A., Aydin C., Arslan N.,Doganalp S., Guney C., Mercan H., Uygur S.O., Uz M. and Yagci O., 2016b, ITU_GRACE16 The global gravity field model including GRACE data up to degree and order 180 of ITU and other collaborating institutions. http://doi.org/10.5880/icgem.2016.006

Barthelmes F., 2013, Definition of Functionals of the Geopotential and Their Calculation from Spherical Harmonic Models: Theory and formulas used by the calculation service of the International Centre for Global Earth Models (ICGEM), Scientific Technical Report STR09/02, Revised Edition, doi:10.2312/GFZ.b103-0902-26.

Barthelmes F. and Köhler W., 2016, International Centre for Global Earth Models (ICGEM), in: Drewes, H., Kuglitsch, F., Adám, J. et al., The Geodesists Handbook 2016, J Geod., 90, 10, 907-1205. doi: 10.1007/s00190-016-0948-z

Bettadpur S., Ries J., Eanes R., Nagel P., Pie N., Poole S., Richter T. and Save H., 2015, Evaluation of the GGM05 Mean Earth Gravity models, Geo. Res. Abs., 17, EGU2015-4153.

Fecher T., Pail R., Gruber T. and the GOCO Project Team., 2016, The Combined Gravity Model GOCO05c. http://doi.org/10.5880/ icgem.2016.003

Förste C. and Bruinsma S.L., 2016, ElGEN-6S4: A time-variable satellite-only gravity field model to d/o 300 based on LAGEOS, GRACE and GOCE data from the collaboration of GFZ Potsdam and GRGS Toulouse. http://doi.org/10.5880/icgem.2016.008

Förste C., Bruinsma S.L., Abrikosov O., Lemoine J. M., Marty J.C., Flechtner F., Balmino G., Barthelmes F. and Biancale R., 2015, EIGEN-6C4 The latest combined global gravity field model including GOCE data up to degree and order 2190 of GFZ Potsdam and GRGS Toulouse. http://dx.doi.org/10.5880/icgem.2015.1

Gatti A., Reguzzoni M., Migliaccio F. and Sanso F., 2014, Space-wise grids of gravity gradients from GOCE data at nominal satellite altitude. [GOCE User Workshop]. Paris

Gilardoni M., Reguzzoni M. and Sampietro D., 2015, GECO: a global gravity model by locally combining GOCE data and EGM2008, Stu. Geo. Geod., doi: 10.1029/2003GL018025.

Huang J. and Véronneau M., 2009, Evaluation of the GRACE-based global gravity models in Canada, N Bull., 4, 3-17.

Mayer-Gurr T., Pail R., Gruber T., Fecher T., Rexer M., Schuh W.D., Kushe J., Brockman J. M., Rieser D., Zehentner N., Kvas A., Klinger B., Baur O., Hock E., Krauss S. and Jaggi, A., 2015, The combined satellite gravity field model GOCO05S. Vienna, Australia.

Morelli C., Gantar C., McConnell R. K., Szabo B., and Uotila U., 1972, The international gravity standardization net 1971 (IGSN 71), Osservatorio Geofisico Sperimentale Trieste (Italy).

Odera P. A., 2016, Assessment of EGM2008 using GPS/leveling and free-air gravity anomalies over Nairobi County and its environs, South African J Geo., 51, 17-30. Doi: http://dx.doi.org/10.4314/sajg.v5i1.2

Osazuwa I.B.,1992a, The Nigerian Standard Calibration Line, Survey Review, 31, 245, 397-408.

Osazuwa I.B.,1985, The Establishment of a Primary Gravity Network for Nigeria, Ph.D. Thesis, Ahmadu Bello University, Zaria, Nigeria.

Ries J., Bettadpur S., Eanes R., Kang Z., Ko, U., McCullough C., Nagel P., Pie, N., Poole S., Richter T., Save H. and Tapley B., 2016, The Combined Gravity Model GGM05C. http://dx.doi.org/10. 5880/icgem.2016.002

Saari T. and Bilker-Koivula M., 2015, Evaluation of GOCE-based Global Geoid Models in Finnish Territory, In EGU General Assembly Conference Abstracts, 17, 4165. 
Šprlák M., Gerlach C., Omang O. and Pettersen B., 2011, Comparison of GOCE derived satellite global gravity models with EGM2008, the OCTAS geoid and terrestrial gravity data: case study for Norway, [Proceedings of the 4th international GOCE user workshop], Munich.

Wenzel H. G., 2005, Global models of the gravity field of high and ultra-high resolution, [International School for the Determination and Use of the Geoid], Budapest, Hungary.
Yılmaz I., Yılmaz M. and Turgut B., 2010, Evaluation of recent global geopotential models based on GPS/levelling data over Afyonkarahisar (Turkey), Sci. Res. and Essays, 5, 5, 484-493.

Zhou H., Luo Z., Zhou Z., Zhong B. and Hsu H., 2016, A new GRACE-only static gravity field model, HUST-Grace2016s. http://doi.org/10.5880/ICGEM.2016.010 